\title{
Evaluación de la calidad de lenguajes de modelado a través de análisis taxonómico: una propuesta preliminar*
}

\author{
Fáber D. Giraldo** \\ Sergio España $a^{* * *}$ \\ William J. Giraldo**** \\ Óscar Pastor ${ }^{* * * *}$
}

Recibido: 14/12/2015 - Aceptado: 02/05/2016

DOI: $10.22395 /$ rium.v15n29a10

\begin{abstract}
Resumen
El paradigma de la ingeniería dirigida por modelos promueve el uso de modelos conceptuales en procesos de ingeniería de sistemas de información. Como productos de ingeniería, los modelos conceptuales deben tener calidad, la cual aplica tanto a los modelos conceptuales como a los lenguajes de modelado empleados para construir dichos modelos. Este artículo presenta un marco de evaluación de la calidad de lenguajes de modelado, en los que se utilizan principios del marco del trabajo Zachman para sistemas de información, como una herramienta taxonómica aplicada sobre artefactos de modelado, empleados en el desarrollo de un sistema de información. El propósito de esta herramienta taxonómica es la ejecución de procedimientos analíticos alineados con una arquitectura de referencia para sistemas de información y razonamientos ontológicos. En este trabajo se expone cómo el marco Zachman soporta análisis sobre lenguajes de modelado para propósitos de calidad por su administración nativa de la semántica.

Palabras clave: calidad, ingeniería dirigida por modelos, sistemas de información, marco de trabajo Zachman, taxonomía, razonamiento ontológico.
\end{abstract}

* Artículo derivado de la investigación "Framework para la evaluación de la calidad en entornos MDE", financiada por COLCIENCIAS entre enero 2012 y marzo 2017. El proyecto fue ejecutado por la Universidad Politècnica de València (España), Universidad del Quindío (Colombia).

** Ing. de sistemas y Computación Universidad del Quindío, magíster en Ingeniería énfasis Informática Universidad EAFIT. PhD(C) en Informática Universidad Politécnica de Valencia (España). Profesor asistente Facultad de Ingeniería Universidad del Quindío, coordinador del énfasis en Ingeniería de Software de la Maestría en Ingeniería de la Universidad del Quindío. Email: fdgiraldo@uniquindio.edu.co. Teléfono: (6) 7359300 ext. 908.

*** Ingeniero informático Universidad Politécnica de Valencia (España). Máster en Ingeniería de Software y Sistemas de Información Universidad Politécnica de Valencia (España). Ph. D en Informática Universidad Politécnica de Valencia (España). Profesor tiempo completo en el Departamento de Informática y Ciencias Computacionales de la Universidad de Utrecht (Holanda).Email: s.espana@uu.nl

***** Ingeniero electricista Universidad del Valle. Máster en Informática Avanzada Universidad Castilla la Mancha (España). PhD en Informática Universidad Castilla la Mancha (España). Profesor asociado Facultad de Ingeniería Universidad del Quindío, Director Grupo de Investigación SINFOCI. Email: wjgiraldo@uniquindio.edu.co. Teléfono: (6)7359300 extensión 995

***** Ph. D en Informática Universidad Politécnica de Valencia (España). Catedrático Departamento de Sistemas Informáticos y Computación (DSIC) Universidad Politécnica de Valencia (España). Director del Centro de Investigación en Métodos de Producción de Software (PROS) de la Universidad Politécnica de Valencia (España). Email: opastor@dsic.upv.es 


\title{
Modelling languages quality evaluation by taxonomic analysis: a preliminary proposal
}

\begin{abstract}
The Model-Driven Engineering (MDE) paradigm promotes the usage of conceptual models in information systems (Is) engineering and research. As engineering products, conceptual models must have quality, which applies on both conceptual models and modeling language employed to build them. This paper presents a modeling language quality evaluation framework. This framework uses the principles from the popular Zachman framework for information systems as a taxonomic tool applied over modeling artifacts used in an information system development. The purpose of this taxonomic tool is to perform analytic procedures that are aligned with an is reference architecture and ontological reasoning. Throughout this work, we describe how the Zachman framework supports analytics over modeling languages for quality purposes by its native management of semantics.
\end{abstract}

Key words: quality, model-driven engineering, information systems, zachman framework, taxonomy, ontological analysis. 


\section{INTRODUCCIÓN}

Los modelos conceptuales son los principales artefactos para controlar la complejidad implícita en los actuales procesos de desarrollo de sistemas de información. Su naturaleza conceptual soporta nativamente las cuestiones derivadas de la presencia de múltiples interesados, niveles de abstracción y retos organizacionales. El paradigma de la ingeniería dirigida por modelos (MDE) hace énfasis en los intereses de negocio y de organización, de manera que los aspectos tecnológicos son resultados de las operaciones sobre los modelos a través de transformaciones o mapeos.

Un importante marco fundacional para trabajar con modelos se propuso en la primera versión de la especificación Model-Driven Architecture (MDA) del Object Management Group (OMG) [1]. En dicha especificación fueron definidos los principios básicos para trabajar y gestionar modelos, resumiéndose estos en dos características principales: la especificación de tres niveles de abstracción (Modelo independiente de Computación - CIM, Modelo Independiente de Plataforma -PIM-, y los Modelos Específicos de Plataforma, PSM), y la definición de las operaciones de transformación de modelos. Sin embargo, el creciente número de practicantes y comunidades MDE, y la falta de un consenso sobre la gestión de modelos (debido principalmente a las divergencias conceptuales de los profesionales) generan desafíos en el uso y gestión de modelos, en donde la especificación MDA 1.0.1 resulta insuficiente. Paradójicamente algunos de los retos derivados son formulados en marcos conceptuales de sistemas de información anteriores a la especificación y publicación oficial de MDA.

Los aspectos más críticos para el paradigma dirigido por modelos radican en los problemas relativos para su adopción en contextos reales. Varios informes indican problemas en la adopción del paradigma relacionados con la falta de alineación entre los principios MDE y el contexto real [2]. Algunas de estas manifestaciones son la sobrecarga impuesta por las herramientas, la falta de mecanismos de trazabilidad, y la falta de apoyo a la adopción de estrategias MDE en procesos organizacionales y/o de desarrollo. En una investigación en curso [3] detectamos otro tipo de problemas reportados en la evaluación de la calidad del MDE, clasificándose en contextos industriales (tales como la insuficiencia de la especificación MDA y problemas en la adopción del paradigma MDE), y los contextos académicos/investigación (difícil operacionalización de los marcos de calidad de MDE, defectos y métricas formuladas principalmente sobre UML, especificidad en escenarios de calidad, y enfoques de calidad de software extrapolados al nivel de modelado).

Evidencias de trabajos basados en modelos y aplicaciones reales sugieren síntomas de evaluación de la calidad respecto a los modelos y lenguajes. En [4] se ha demostrado la significativa divergencia de la concepción de calidad para MDE. La guía MDA 
2.0 [5] publicada en junio de 2014 intenta hacer frente a los actuales desafíos de los modelos conceptuales, incluida la evaluación de la calidad de los modelos mediante análisis de datos semánticos extraídos desde modelos (analítica de modelos en MDA 2.0). Sin embargo, esta especificación no prescribe cómo realizar este tipo de análisis y evaluación de la calidad de los modelos y lenguajes.

En este trabajo se propone una taxonomía basada en el marco de trabajo Zachman $[6,7]$ para el análisis de la calidad en MDE. Creemos que el paradigma dirigido por modelos y los principios de sistemas de información recopilados por el marco Zachman tienen una fuerte correlación, y dicho marco puede apoyar la evaluación de los desafíos actuales de calidad del MDE. El resto de este documento se estructura de la siguiente manera: la sección 2 presenta manifestaciones actuales de calidad en MDE y algunos enfoques para su tratamiento. La sección 3 expone el soporte conceptual para considerar Zachman como una taxonomía MDE. La sección 4 presenta algunos procedimientos analíticos preliminares derivados de la taxonomía Zachman aplicada sobre artefactos de modelado. La sección 5 presenta la justificación de nuestra propuesta, basada en un contraste de la taxonomía Zachman respecto a métodos de análisis ontológicos y evaluación de taxonomías. Finalmente, se concluye el documento.

\section{MANIFESTACIONES DE CALIDAD EN MDE Y SU TRATAMIENTO TÍPICO}

El paradigma dirigido por modelos no tiene una concepción común y, en su lugar, nos encontramos con una plétora de interpretaciones de acuerdo con los objetivos de cada comunidad MDE. La referencia más neutral y aceptada para MDE es la especificación MDA que refleja la visión particular de la OMG (Object Management Group) sobre esta disciplina. La guía MDA sirve como una referencia común para considerar roles y operaciones sobre modelos. Aunque la guía MDA 1.0.1 [1] fue una especificación clave para el MDE, su falta de actualizaciones durante una década contribuyó a la aparición de nuevos retos para los profesionales MDE, cada uno dirigido desde esfuerzos e iniciativas particulares.

Claramente, un intento de hacer frente a los nuevos desafíos implícitos en la tarea de modelado se evidencia en la guía MDA 2.0 [5]. Sin embargo, este esfuerzo no es suficiente teniendo en cuenta que i) la guía MDA carece de especificaciones acerca de cómo identificar y gestionar los datos semánticos derivados de modelos, incluyendo los aspectos de su uso previsto más allá de la aplicación de estándares MDE. ii) La mayoría de los desafíos actuales para el paradigma dirigido por modelos se han formulado desde marcos conceptuales previos para sistemas de información, tales como FRISCO [8] (diciembre de 1996) en donde se definen los aspectos clave para el paradigma: el uso de los modelos (modelado conceptual) en sí mismo, la definición de los 
sistemas de información y el uso de notaciones de sistemas de información mediante representaciones (modelos), la definición de sistema de información computarizados y el nivel de abstracción cero originado en la presencia de los procesadores.

\subsection{Análisis ontológico/taxonómico}

Una técnica para enfrentar los desafíos del paradigma dirigido por modelos es el análisis ontológico, es decir, la evaluación de los elementos de los lenguajes de modelado, elementos con respecto a la orientación proporcionada por marcos ontológicos para sistemas de información. Aunque la pregunta sobre si la orientación ontológica resulta en mejores modelos es una cuestión abierta [9], se emplean comúnmente ontologías para sistemas de información como BWW, UFO, CORE, Dolce y similares para evaluar lenguajes de modelado con respecto a construcciones ontológicas, estableciendo su integridad y claridad a través de un proceso de mapeo entre constructos ontológicos y de modelado.

El principal soporte proporcionado por el análisis ontológico es el razonamiento basado en inferencias, donde el analista/diseñador de lenguajes puede evaluar las implicaciones de las constructos de los lenguajes con respecto a alguna representación específica del mundo real de interés para los usuarios de sistemas de información. De acuerdo con [10], si el modelo es ontológicamente incompleto, el analista/diseñador de alguna manera tiene que aumentar el (los) modelo(s) para asegurar que el sistema de información computarizado resultante refleja adecuadamente esa parte del mundo real que se pretende simular. Con frecuencia la calidad se refiere a soluciones basadas en ontologías debido a la dificultad de distinguir entre el espacio del problema y el espacio de la solución, alcances estos asociados a los enfoques dirigidos por modelos y basados en ontologías, respectivamente.

La particularidad en el análisis proporcionado por ontologías de sistemas de información contribuye con las divergencias conceptuales en la comunidad MDE y la consolidación de iniciativas aisladas de sistemas de información con sus comunidades asociadas. La suficiencia filosófica de las ontologías no se cuestiona en sí misma. Sin embargo, si los lenguajes de modelado deben enfrentarse a su particular evaluación a través de múltiples marcos ontológicos probablemente estas actividades conducirán a sobrecargas en la formulación y evaluación de lenguajes, debido a que la coherencia entre los resultados de los múltiples análisis ontológicos no está garantizada, es decir, para el mismo lenguaje se podrían obtener varios resultados en sus evaluaciones de acuerdo con las concepciones particulares de cada marco ontológico.

Otra herramienta conceptual reportada en procesos analíticos sobre contextos dirigidos por modelos es el análisis taxonómico. Esta herramienta sirve de guía para las

Revista Ingenierías Universidad de Medellín, vol. 15, №. 29 pp. 159-172 ISSN 1692-3324 - julio-diciembre de 2016/208 p. Medellín, Colombia 
tareas específicas de modelado mediante la clasificación de los artefactos y/o técnicas. Las decisiones sobre los artefactos de modelado se hacen basándose en un esquema de clasificación anterior.

A pesar del alto potencial del análisis taxonómico para la comprensión de las implicaciones de las prácticas MDE se reporta una baja tasa de informes relacionados con su aplicación y uso. También, en forma similar al análisis ontológico, el proceso de evaluación taxonómica puede conducir a un análisis subjetivo debido a su enfoque sobre características específicas del paradigma MDE. Ambas técnicas de análisis ontológico y taxonómico se deben utilizar de forma complementaria. La inferencia y el mecanismo de clasificación son estrategias valiosas para manejar los desafíos en la propia adopción del MDE por su apoyo a los razonamientos sobre los modelos.

\section{UNA TAXONOMÍA DE REFERENCIA PARA DESCUBRIR ASPECTOS DE CALIDAD EN MDE}

Independientemente de marcas comerciales el marco Zachman [6,7] es una taxonomía de referencia derivada desde fuentes lingüísticas y filosóficas, cuyo objetivo principal es apoyar un proceso racional sobre el uso sistémico de elementos dentro de un sistema de información, con el fin de definir una solución empresarial. Este marco fue concebido como una propuesta de arquitectura para la descripción de un sistema de información, identificando los elementos esenciales que integran un sistema holístico y que se desplegarán en una organización. El marco ha establecido las bases para estándares críticos actuales como ISO 42010 [11] (descripciones arquitectónicas de software y sistemas) y los marcos actuales de arquitectura empresarial.

La característica principal del marco Zachman es su soporte nativo para la gestión semántica a través de su estructura bidimensional semántica donde los modelos conceptuales involucrados en un proceso de desarrollo de sistemas de información se pueden clasificar. La guía MDA 2.0 hace especial énfasis en los datos semánticos para ejecutar procesos analíticos sobre modelos. Sin embargo, esta no define ningún método para deducir este tipo de datos a través de artefactos de modelado. La semántica se define en términos de un dominio semántico y unasintaxis de mapeo dentro de ese dominio. El dominio semántico especifica los conceptos que existen en el universo del discurso, y es un requisito previo para la comparación de las definiciones semánticas [12]. La taxonomía Zachman ofrece un dominio semántico que abstrae la realidad de un sistema de información, permitiendo capturar las decisiones sobre el tipo de expresiones que los artefactos de modelado deben soportar. Esto implica que el significado del artefacto de modelado es reconocido para fines de análisis.

La taxonomía Zachman por sí misma no define ningún tipo de relación entre los elementos del marco. Solo define reglas para clasificar la información derivada de los 
modelos conceptuales. De esta manera, cualquier análisis ontológico por inferencia se deriva del contraste entre la información acerca de los modelos conceptuales empleados frente a fenómenos esenciales (o clasificadores) proporcionados por el marco en sí mismo (cosas, procesos, lugares, personas, eventos y estrategias). La taxonomía Zachman y los marcos ontológicos para sistemas de información son herramientas filosóficas complementarias por la presencia común de una definición de arquitectura para sistemas de información.

La contribución más relevante de la taxonomía Zachman para contextos MDE es el soporte al razonamiento sobre modelos y lenguajes de modelado en sistemas de información, es decir, la justificación subyacente para la creación y el uso de artefactos de modelados relacionados. Los diseñadores de lenguajes de modelado tienen una herramienta para toma de decisiones que permite justificar el propósito y las intenciones de los esfuerzos particulares de modelado, teniendo en cuenta la cantidad actual de los métodos para sistemas de información. Esto afecta directamente la armonización de lenguajes de modelado. En cuanto a otras propuestas taxonomías para iniciativas MDE, Zachman brinda un alcance taxonómico más completo, ya que considera los puntos de vista (y sus vistas resultantes) directamente asociadas a contextos organizacionales (desde nivel de negocio hasta nivel técnico), donde será desplegado un sistema de información. Las operaciones sobre modelos (transformaciones) son resultado de la interacción de los modelos dentro de los niveles del sistema de información.

\subsection{Soporte del marco Zachman para las manifestaciones de calidad en MDE}

Desde su formulación, la taxonomía Zachman trabaja con modelos conceptuales para representar fenómenos de un sistema de información, resultantes de combinar abstracciones con puntos de vista. El objetivo principal de esta herramienta es relacionar elementos conceptuales con las representaciones computarizadas [7]. La taxonomía en sí no propone ningún procedimiento especial o metodología para evaluar los modelos y lenguajes. Sin embargo, debido a este apoyo nativo para el modelado, los actuales problemas de calidad sobre lenguajes y modelos pueden ser abordados por análisis y procedimientos alineados con la propia actividad de la clasificación. Algunos ejemplos de manifestaciones de calidad que podrían abordarse en el marco son:

- La separación de intereses: la taxonomía promueve la selección de subconjuntos sobre los cuales se aplican decisiones. En lugar de administrar la complejidad mediante el modelo general de todas las celdas del marco, la taxonomía sugiere que las decisiones sean tomadas con respecto al alcance de cada celda. Además, la recursividad y la lógica del marco permiten describir cualquier atributo específico cuya complejidad no pudiera ser manejada adecuadamente en una clasificación 
global donde se analizan simultáneamente múltiples propiedades. Las descripciones están alineadas con el contexto original de un sistema de información.

- Comunicación e idoneidad: el fundamento de la taxonomía es la existencia de un conjunto de representaciones arquitectónicas aditivas y complementarias para sistemas de información. Este es un tema crítico: en el documento del modelo fundacional del MDA [1] la metaclase modelo se asocia explícitamente con el término descripción arquitectónica definido en la norma ISO 42010. De esta manera, un mecanismo de razonamiento es necesario para discernir sobre la selección de una representación arquitectónica específica para una combinación abstracción/perspectiva, teniendo en cuenta la variedad de notaciones, metodologías y herramientas relacionadas.

- Capacidades para la integración de modelos: la coexistencia de múltiples iniciativas de modelado debe ser evaluada para desarrollar un sistema de información de manera coherente y optimizada. Esto implica el análisis sobre las capacidades ofrecidas por los lenguajes de modelado para abordar cuestiones relacionadas con cada celda de la taxonomía, en función del (de los) objetivo(s) principal(es) del propio lenguaje de modelado y sus artefactos provistos. Este contraste de todos los lenguajes de modelado implicados en un proyecto de desarrollo de sistema de información con el marco taxonómico podría mejorar esfuerzos de modelado, evitando la duplicación de técnicas de modelado (es decir, el uso de múltiples modelos en diferentes lenguajes de modelado para representar la misma información), la identificación de aspectos complementarios según los requisitos específicos de las técnicas de modelado, y la identificación de intereses de sistemas de información no cubiertos por los lenguajes empleados.

- Transformaciones de modelos: uno de los retos más importantes formulados por el marco es garantizar que las transformaciones de modelos tengan lugar como una directa consecuencia de la adición de información con respecto a la interacción con abstracciones y perspectivas. La función de mapeo de modelos (mencionada en la guía original de MDA) se produce de forma nativa durante los cambios estructurales en los modelos que se atraviesan de perspectivas superiores a inferiores. La información al nivel CIM se enriquece con restricciones asociadas a los niveles más bajos, obteniendo así la información suficiente para facilitar su aplicación en un entorno técnico. De forma similar, la transformación entre la información en dos columnas debe ser lo suficientemente justificada para soportar los criterios del diseñador del lenguaje de derivar modelos asociados a diferentes propiedades esenciales, por ejemplo, de tiempo a localización, o de proceso a datos. Las evidencias de trazabilidad se pueden derivar desde análisis sobre la información clasificada por el marco. 
La estructura taxonómica de la propuesta Zachman proporciona un conjunto útil y valioso de información necesaria para modelar cualquier fenómeno dentro del ámbito de aplicación de un sistema de información [15]. Adicionalmente, las reglas de clasificación garantizan la coherencia para cualquier actividad de modelado en un sistema de información.

\subsection{Procedimientos preliminares de evaluación de calidad basados en clasificación}

La clasificación es el propósito principal del marco Zachman, independientemente de sus usos comerciales derivados. La taxonomía basada en Zachman define clasificadores, es decir, los elementos esenciales de cada celda (en cada fila/columna), necesarios para concebir y producir un modelo a partir de una combinación de nivel de abstracción/ vista. Los puntos de vista de modelado son compatibles con los fundamentos de modelado conceptual relacionados en [13,14]. La ingeniería dirigida por modelos cubre todos los dominios de la arquitectura de un sistema de información definidos por el marco Zachman.

En [15] se propone una articulación sistémica entre MDA (base arquitectónica para el uso y transformaciones de modelos) y Zachman. MDA apoya Zachman explícitamente por la definición de los niveles de abstracción y fundamentos de mapeo a través de las relaciones superior-inferior entre niveles. Zachman complementa MDA por la definición de las dimensiones de modelado que soportan el mapeo y transformación de modelos a niveles conceptuales, independiente de su implementación asociada sobre un lenguaje específico para transformación de modelos.

La figura 1 expone preliminarmente algunos procedimientos de evaluación de la calidad de modelado que se pueden realizar con la taxonomía Zachman. Otras ventajas de este enfoque son las siguientes:

- El marco Zachman es compatible con marcos ontológicos y estándares para sistemas de información.

- Se proporciona un apoyo visual explícito y estandarizado para analizar iniciativas de modelado.

- La evaluación analítica con el marco Zachman es relativamente similar a una evaluación ontológica, pero en lugar de utilizar las concepciones particulares de dominios, se emplea una arquitectura universal para sistemas de información ampliamente aceptada incluso por los estándares más representativos de esta área de conocimiento. 


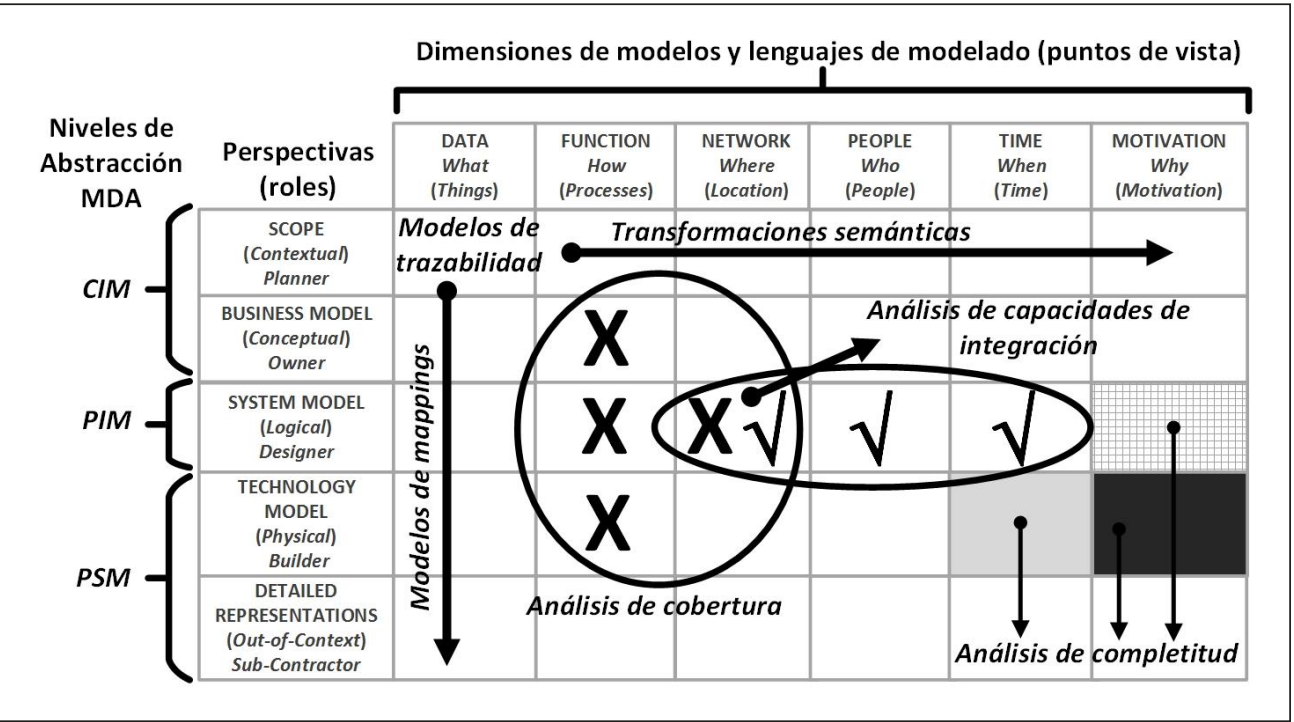

Figura 1. Resumen de la aplicabilidad de la taxonomía Zachman para la evaluación de la calidad en contextos MDE Fuente: $[18,19]$

- Esta técnica soporta conceptual y metodológicamente la evaluación de la idoneidad de lenguajes de modelado en cuanto a su propósito, la cobertura identificada, y los niveles de abstracción / las dimensiones de modelado involucrados.

- El marco permite contrastar la información suministrada por los diagramas (consecuencia natural de las notaciones y semánticas aplicadas) con respecto a las intenciones de modelado.

- A través del marco se pueden identificar las capacidades de integración que ofrecen los lenguajes de modelado. Por ejemplo, los autores en [16] utilizan Zachman como un conjunto de capas para definir elementos interrelacionados, y establecer la coherencia en la aplicación de dos lenguajes de modelado para apoyar a grupos multidisciplinarios en el diseño y desarrollo de sistemas interactivos para el trabajo en grupo.

- El marco provee un soporte nativo para mapeos, transformaciones y gestión de la trazabilidad. El marco hace explícita la naturaleza del mapeo y las especificaciones para los procesos de transformación.

- Como una herramienta conceptual el marco proporciona un repositorio de conocimiento compartido para el razonamiento, análisis y comunicación sobre modelos, lenguajes, y sus implicaciones en un proyecto real de sistemas de información. 
La clasificación en sí misma exige la consideración explícita de aspectos técnicos de la implementación de los artefactos de modelado bajo análisis. Es criterio del analista/diseñador decidir si se consideran o no los aspectos técnicos. Sin embargo, la implementación funcional debe ser considerada en algún momento, para evidenciar la viabilidad del esfuerzo de modelado desde una perspectiva computacional.

\section{VALIDACIÓN TEÓRICA}

La característica clave del marco Zachman es la clasificación de los artefactos de modelado que se ajustan entre sí de una forma sistémica. Para estas tareas, y el consiguiente apoyo al análisis ontológico, la suficiencia de la taxonomía debe ser evaluada a través de mecanismos derivados desde ontologías formales para el análisis de taxonomías. En este trabajo se sigue un procedimiento de evaluación ontológica [17] mediante coincidencia de constructos de métodos previos para sistemas de información, y la metodología expuesta en $[18,19]$, de manera que preliminarmente se analiza la taxonomía Zachman con respecto a las nociones esenciales que debe cumplir cualquier taxonomía bien formulada.

La metodología formulada en [18, 19] define la taxonomía Backbone. Se trata de un conjunto de propiedades especiales asociadas a una taxonomía, que permiten establecer una estructura sobre una ontología. La taxonomía Backbone está compuesta por:

- Formal roles: son propiedades que expresan el papel de una entidad en un evento, a menudo ejemplificando una relación particular entre dos o más entidades.

- Material roles: estos son elementos que heredan condiciones de identidad de algún tipo. Ellos representan los roles que están restringido a determinados tipos de entidades.

- Phased Sortals, que corresponden a una cierta fase temporal de sus instancias.

- Attributions: estos representan valores de atributos (o cualidades) como color, forma, etc.

- Mixins: estas propiedades representan intuitivamente varias combinaciones (disyunciones o conjunciones).

La figura 2 expone el mapeo de la taxonomía Zachman con la taxonomía Backbone. Para este caso, los formal roles se asignan a los roles asociados a las perspectivas. Los material roles son aquellos que participan en el acto mismo de modelado (ya sea en el modelado o en la definición/soporte a un lenguaje de modelado), como experto en negocios, analista de sistemas, diseñador, arquitecto, desarrollador, etnógrafo, experto 


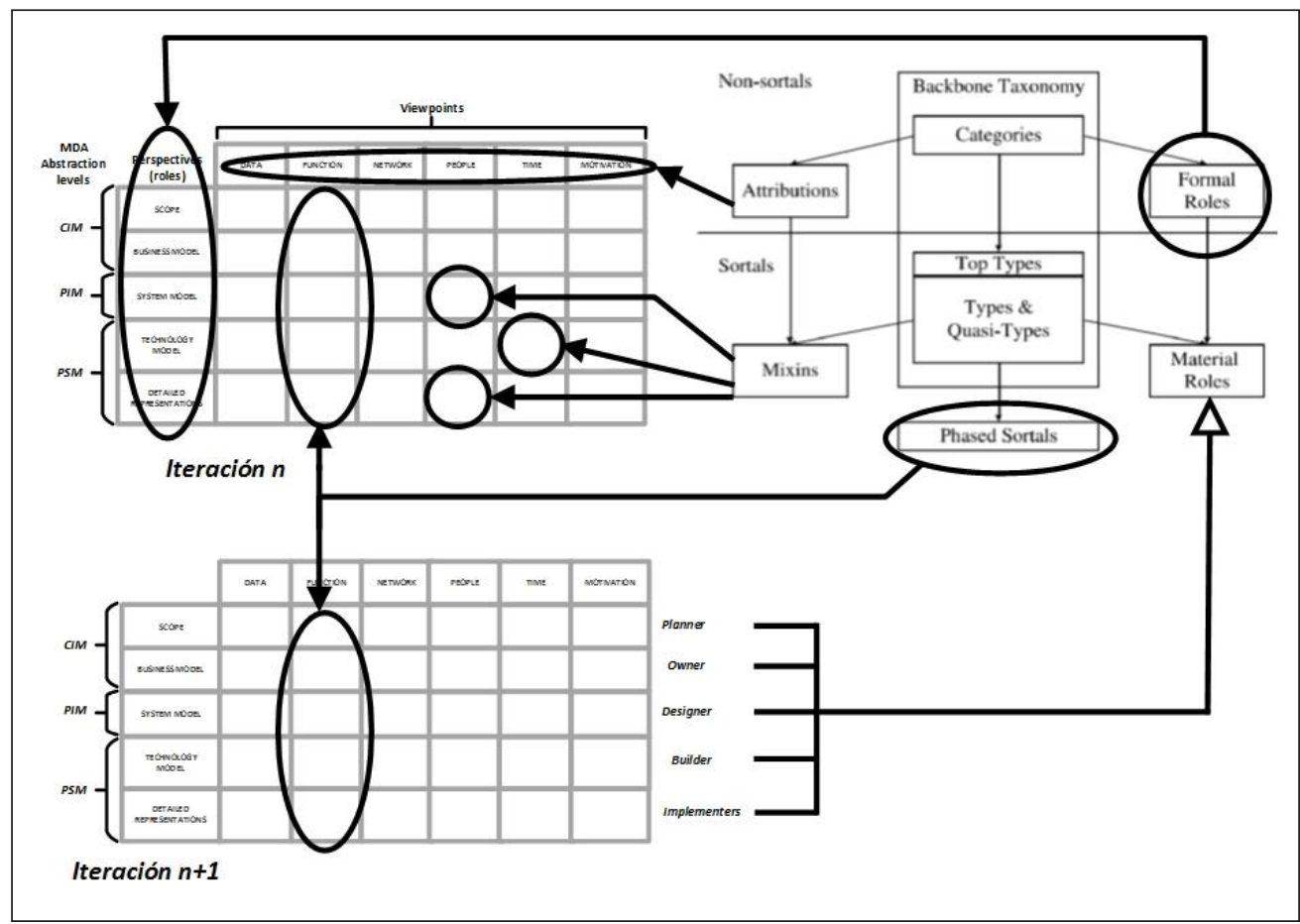

Figura 2. Mapeo de la taxonomía Zachman con la taxonomía de referencia Backbone Fuente: $[18,19]$

en seguridad y otros. Estos son definidos como roles asociados a puntos de vista que heredan atributos desde los roles de las perspectivas nativas Zachman. Las phased sortals se refieren a los diferentes tiempos en los que se puede realizar el análisis taxonómico. Para este caso, tenemos análisis temporal cuando los modelos se cruzan entre los niveles de abstracción CIM-PIM-PSM. Otros phased sortal ocurren cuando el marco se aplica en etapas posteriores del proceso de modelado en el tiempo, por ejemplo, para comprobar el progreso del modelado y desarrollo del sistema de información. Las attributions son definidas por el modelo esencial de cada punto de vista y la coherencia semántica de la cada abstracción / perspectiva. Ambos elementos prescriben los atributos de los artefactos de modelado que se encuentran bajo clasificación. Los mixins son la combinación de abstracción / perspectiva y puntos de vista propiedades en forma de celdas.

\section{CONCLUSIONES}

En este trabajo se propuso preliminarmente una taxonomía para la identificación y evaluación de los retos de calidad en MDE. Este enfoque aprovecha los principios esenciales del marco Zachman para estructurar iniciativas MDE sobre sistemas de 
información a través de la clasificación. Este análisis taxonómico ayuda a resolver la pregunta sobre cuándo una iniciativa de modelado es compatible con MDE. Los modelos conceptuales utilizados deben manifestar su soporte mediante la definición de los elementos básicos del lenguaje, la presencia de algún mecanismo de representación, así como la alineación con intereses de sistemas de información. Además, este enfoque apoya el análisis ontológico por los razonamientos derivados desde la actividad de clasificación.

\section{AGRADECIMIENTOS}

F.D. Giraldo agradece al Departamento Administrativo de Ciencias, Tecnología e Innovación -COLCIENCIAS- por la financiación de este proyecto a través de la convocatoria 512/2010.

\section{REFERENCIAS}

[1] OMG, "MDA Guide Version 1.0.1," http://www.omg.org/cgibin/doc?omg/03-06-01.pdf, Jun. 2003. [Online]. Available: http://www.omg.org/cgi-bin/doc?omg/03-06-01.pdf

[2] J. Whittle, J. Hutchinson, and M. Rouncefield, "The state of practice in model-driven engineering”, Software, IEEE, vol. 31, N. 3, pp. 79-85, May 2014.

[3] Giraldo, Fáber D.; España, Sergio; Giraldo, William J.; Pastor, Óscar, "Modelling language quality evaluation in model-driven information systems engineering: A roadmap", Research Challenges in Information Science (RCIS), 2015 IEEE 9th International Conference on, vol., N. ${ }^{\circ}$, pp. 64,69, 13-15 May 2015 doi: 10.1109/RCIS.2015.7128864.

[4] F. Giraldo, S. España, and O. Pastor, "Analysing the concept of quality in model-driven engineering literature: A systematic review", in Research Challenges in Information Science (RCIS), 2014 IEEE Eighth International Conference on, May 2014, pp. 1-12.

[5] OMG, "MDA Guide revision 2.0", http://www.omg.org/cgibin/doc?ormsc/14-06-01.pdf, Jun. 2014. [Online]. Available: http://www.omg.org/cgi-bin/doc?ormsc/14-06-01.pdf

[6] J. A. Zachman, "A framework for information systems architecture", IBM Syst. J., vol. 26, N. 3, pp. 276-292, Sep. 1987. [Online]. Available: http://dx.doi.org/10.1147/sj.263.0276

[7] J. F. Sowa and J. A. Zachman. Extending and formalizing the framework for information systems architecture. IBM Syst. J., 31(3):590\{616, June 1992.

[8] E. D. Falkenberg, W. Hesse, P. Lindgreen, B. E. Nilsson, J. L. H. Oei, C. Rolland, R. K. Stamper, F. J. M. V. Assche, A. A. Verrijn-Stuart, and K. Voss, "Frisco: A framework of information system concepts", The IFIP WG 8. 1 Task Group FRISCO, Tech. Rep., 1996. [Online]. Available: http://cs-exhibitions.uni-klu.ac.at/index.php?id=445

[9] A. Saghafi and Y. Wand, "Do ontological guidelines improve understandability of conceptual models? a meta-analysis of empirical work", in System Sciences (HICSS), 2014 47th Hawaii International Conference on, Jan 2014, pp. 4609-4618. 
[10] M. Rosemann and P. Green, "Integrating multi-perspective views into ontological analysis", in Proceedings of the Twenty First International Conference on Information Systems, ser. ICIS'00, 2000, pp. 618-627. [Online]. Available: http://dl.acm.org/citation.cfm?id=359640.359915

[11] ISO/IEC/IEEE systems and software engineering-architecture description", ISO/IEC/IEEE 42010:2011(E) (Revision of ISO/IEC 42010:2007 and IEEE Std 1471-2000), pp. 1-46, Dec 2011.

[12] D. Harel and B. Rumpe, "Meaningful modeling: What's the semantics of "semantics"?" Computer, vol. 37, N. ${ }^{\circ}$ 10, pp. 64-72, Oct. 2004. [Online]. Available: http://dx.doi.org/10.1109/ MC.2004.172

[13] Bernhard Thalheim. The theory of conceptual models, the theory of conceptual modelling and foundations of conceptual modelling. In David W. Embley and Bernhard Thalheim, editors, Handbook of Conceptual Modeling, pages 543-577.Springer Berlin Heidelberg, 2011.

[14] Bernhard Thalheim. Syntax, semantics and pragmatics of conceptual modelling. In Gosse Bouma, Ashwin Ittoo, Elisabeth Mtais, and Hans Wortmann, editors, Natural Language Processing and Information Systems, volume 7337 of Lecture Notes in Computer Science, pages 1\{10. Springer Berlin Heidelberg, 2012.

[15] David S. Frankel, et al. The Zachman Framework and the OMG Model Driven Architecture, September 2003.

[16] Ana I. Molina, William J. Giraldo, Manuel Ortega, Miguel A. Redondo, and César A. Collazos. Model-driven development of interactive groupware systems: Integration into the software development process. Science of Computer Programming, 89, Part C(0):320-349, 2014.

[17] Keng Siau and Matti Rossi. Evaluation of information modeling methods - a review. In HICSS (5), pages $314\{322,1998$.

[18] N. Guarino and C. A. Welty, "A formal ontology of properties", in Proceedings of the 12th European Workshop on Knowledge Acquisition, Modeling and Management, ser. EKAW '00. London, UK, UK: Springer-Verlag, 2000, pp. 97-112. [Online]. Available: http://dl.acm.org/ citation.cfm?id $=645361.650850$

[19] C.Welty and N. Guarino, "Supporting ontological analysis of taxonomic relationships," Data Knowl. Eng., vol. 39, N. ${ }^{\circ}$ 1, pp. 51-74, Oct. 2001. [Online]. Available: http://dx.doi.org/10.1016/ S0169-023X (01)00030-1 